\title{
Influência de fatores abióticos na eclosão de ovos dormentes do rotífero Brachionus calyciflorus Pallas (Monogononta, Ploima)
}

\author{
Nilton Eduardo Torres Rojas 1, 3 \\ José Roberto Verani ${ }^{2}$ \\ Maria Amália Basile-Martins ${ }^{1}$
}

\begin{abstract}
The effect of abiotic factors on the hatching of Brachionus calyciflorus Pallas (Monogononta, Ploima) resting eggs. The factors involved in the induction of resting eggs development and hatching are poorly understood for Brachionus calyciflorus populations of South Hemisphere. The role of some abiotic factors in controlling the hatching rates of $B$. calyciflorus resting eggs was investigated. Determination of optimum hatching conditions would be important in developing the use of this species as food for fish larvae in aquaculture. Resting eggs were exposed to different treatments in the laboratory, and monitored for hatching over a period of five days. Optimum hatching conditions were: $\mathrm{pH} 5-9$, water ionic concentrations from 220 to $880 \mathrm{mg} . \mathrm{I}^{-1}$ of selected salts, temperature $25-30^{\circ} \mathrm{C}$, photoperiod eight or more hours light per day, and light intensity equal to or greater than 850 lux.

KEY WORDS. Brachionus calyciflorus, rotifer, resting eggs, hatching eggs, abiotic factors
\end{abstract}

A diversidade de tipos de reprodução, que variam da partenogênese à reprodução sexuada, bem como a produção de ovos de repouso, são os aspectos biológicos mais marcantes do ciclo de vida de alguns organismos zooplanctônicos. Os ovos dormentes, ou embriões encistados, que podem permanecer em repouso por longos períodos, são estudados por pesquisadores que procuram entender seu significado ecológico e as características biológicas da espécie que os produz (GILBERT 1974; King 1980; POURRIOT \& SNELl 1983). Os ovos dormentes, ou embriões encistados, que podem permanecer em repouso por longos períodos, são estudados por pesquisadores que procuram entender seu significado ecológico e as características biológicas da espécie que os produz (GILBERT 1974; KING 1980; POURRIOT \& SNELL 1983), e sua produção em massa para utilização na aqüicultura (HAGIWARA (1994).

Os fatores que determinam a interrupção do repouso e a eclosão de ovos dormentes ainda necessitam ser melhor investigados, principalmente se levar em consideração as diferentes linhagens zoogeográficas. Portanto, o objetivo deste trabalho foi verificar se há qualquer relação entre $\mathrm{pH}$, concentração de íons dissol-

1) Instituto de Pesca. Avenida Francisco Matarazzo 455, 05001-900 São Paulo, São Paulo, Brasil.

2) Departamento de Hidrobiologia, Universidade Federal de São Carlos. Estrada Washington Luis, Km 235, 13565-905 São Carlos, São Paulo, Brasil.

3) Bolsista da CAPES. 
vidos, temperatura, fotoperíodo e intensidade luminosa e a eclosão de ovos dormentes de uma população do rotífero Brachionus calyciflorus Pallas, 1776, procurando subsidiar a definição de técnicas de eclosão.

\section{MATERIAL E MÉTODOS}

Os rotíferos foram coletados em viveiros de criação de peixes, em Pirassununga ( $\left.21^{\circ} 58^{\prime} \mathrm{S}, 47^{\circ} 26^{\prime} \mathrm{W}\right)$, São Paulo, Brasil.

As populações foram mantidas em aquários contendo 30 a 40 litros de água, instalados em laboratório (climatizado à temperatura de $25 \pm 1^{\circ} \mathrm{C}$, fotoperíodo de 14 horas e intensidade luminosa de 500 lux com lâmpada fluorescente). Semanalmente, dois terços da água dos recipientes de cultivo era renovada. A água reposta provinha de uma caixa de fibro-cimento (mil litros de capacidade) colocada externamente ao laboratório e contendo plantas aquáticas (Elodea sp.) e peixes (Poecilia sp.). O nível da água desta caixa era mantido principalmente com água pluvial. Antes de ser utilizada nos recipientes de cultivo, a água era filtrada mecanicamente em rede de plâncton de $30 \mu \mathrm{m}$ e aerada por 24 horas.

Os rotíferos, alimentados "ad libitum" duas vezes ao dia, recebiam, pela manhã, levedura de panificação (Saccharomyces cerevisiae) e, ao final da tarde, microalgas (Chlorella homosphaera e/ou Scenedesmus ecornis e/ou Kirchneriella lunaris e/ou Chlamydomonas sp. e/ou Nephrocylium lunatium) cultivadas em meio de cultura W.C. (GUILLARD \& LORENZEN 1972) e centrifugadas durante a fase exponencial de crescimento antes de serem utilizadas. Para produção das algas foi utilizada metodologia semelhante à descrita por PoRTElla et al. (1997), que

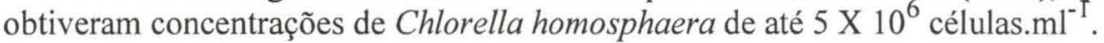

A produção de ovos dormentes pelas populações de B. calyciflorus, cultivadas em laboratório, foi espontânea. Semanalmente, o fundo dos recipientes de cultivo era inspecionado e, ao se constatar a presença de um número suficiente de ovos para montagem de um experimento, estes eram coletados por sifonagem e mantidos em água à temperatura de $5 \pm 1^{\circ} \mathrm{C}$, em um refrigerador. Após um período mínimo de 15 dias, os ovos eram testados para verificar a ocorrência ou não de eclosão (perda de dormência) e, posteriormente, serem utilizados nos testes.

Os experimentos, com duração de cinco dias, foram realizados em vidros de relógio ( $7 \mathrm{~cm}$ de diâmetro) contendo $7 \mathrm{ml}$ de água.

Para os testes de eclosão de ovos nos experimentos 1, 3, 4 e 5 foi utilizada uma mistura de água do filtro biológico e água destilada (1:1), previamente aerada por 12 horas. Para o preparo das soluções do experimento 2 foi utilizada água deionizada, também aerada pelo mesmo período, antes do experimento.

Durante os experimentos a água era trocada diariamente para monitoramento ou correção do $\mathrm{pH}$ e da condutividade.

No primeiro experimento os ovos foram expostos aos valores de $\mathrm{pH} 3$ a 12 . Para ajuste do $\mathrm{pH}$ foram utilizadas soluções de hidróxido de sódio $(\mathrm{NaOH})$ e de ácido clorídrico $(\mathrm{HCl})$.

No segundo experimento os ovos foram mantidos em meios contendo diferentes concentrações dos principais íons que ocorrem no ambiente aquático. A composição destes meios é apresentada em AMERICAN Public Health AssociaTION (1989) como apropriada para ensaios de laboratório (Tab. I). 
Tabela I. Tipos e composição dos sais empregados no experimento 2. Além destes tratamentos, foi aplicado outro com água deionizada.

\begin{tabular}{lcccr}
\hline \multirow{2}{*}{ Tipo de água } & \multicolumn{4}{c}{ Concentraçăo de sais utilizada $\left(\mathrm{mg} \mathrm{I}^{-1}\right)$} \\
\cline { 2 - 5 } & $\mathrm{NaHCO}_{3}$ & $\mathrm{CaSO}_{4} .2 \mathrm{H}_{2} \mathrm{O}$ & $\mathrm{MgSO}_{4}$ & $\mathrm{KCl}$ \\
\hline Muito mole & 12,0 & 7,5 & 7,5 & 0,5 \\
Mole & 48,0 & 30,0 & 30,0 & 2,0 \\
Moderadamente dura & 96,0 & 60,0 & 60,0 & 4,0 \\
Dura & 192,0 & 120,0 & 120,0 & 8,0 \\
Muito dura & 384,0 & 240,0 & 240,0 & 16,0 \\
\hline
\end{tabular}

No terceiro experimento, realizado em câmara de germinação, os ovos foram mantidos nas temperaturas de $10,15,20,25,30,35$ e $40 \pm 1^{\circ} \mathrm{C}$. A intensidade luminosa no interior da câmara (650 lux com lâmpada fluorescente) foi semelhante à utilizada no laboratório para a realização dos experimentos 1 e 2 .

No quarto experimento os ovos foram submetidos, na câmara de germinação, a diferentes fotoperíodos: $0,8,12,16 \mathrm{e} 24$ horas. A temperatura utilizada foi $25 \pm 1^{\circ} \mathrm{C}$ e a intensidade luminosa, quando necessária, 650 lux.

No quinto experimento, realizado em câmara de germinação, os ovos foram submetidos a diferentes intensidades luminosas: 0, 650, 850, 1350 e 2000 lux. A temperatura foi mantida em $25 \pm 1^{\circ} \mathrm{C}$ e o fotoperíodo foi de 12 horas, com exceção do tratamento em que os ovos permaneceram no escuro.

Para cada experimento e/ou réplica todos os ovos utilizados, foram produzidos por uma mesma população e em uma mesma época.

Cada experimento teve uma réplica. Cada tratamento de cada experimento teve 10 repetições, utilizando-se 10 ovos em cada uma. Portanto, para cada tratamento de cada experimento foram feitas 20 repetições com total de 200 ovos. Para todos os experimentos foram utilizados 6600 ovos.

Para verificação do efeito dos fatores estudados na taxa de eclosão dos ovos, aplicou-se a Análise de Variância (ANOVA) para experimentos inteiramente casualizados, considerando-se as 20 repetições de cada tratamento em nível de confiança de $95 \%$. Como complementação a esta análise, nos casos em que o resultado foi significativo aplicou-se o teste de Tukey $(\mathrm{p}<0,05)$, para agrupamento de médias significativamente iguais (SNEDECOR \& COCHRAN 1971).

\section{RESULTADOS}

Os ovos dormentes de $B$. calyciflorus apresentaram tamanho médio de $166,20 \pm 15,51$ X 111,56 $\pm 11,86 \mu \mathrm{m}$; para $\mathrm{n}=100$.

Os resultados obtidos com os ovos dormentes de B. calyciflorus expostos a diferentes valores de $\mathrm{pH}$ indicam que eles eclodem em uma ampla variação deste fator (Fig. 1). Observa-se que não existem diferenças significativas entre os resultados obtidos em valores de $\mathrm{pH}$ de 5 a $9(\Delta$ Tukey $=1,596 ; \mathrm{p}<0,05)$. Fora desta faixa de $\mathrm{pH}$ também ocorreu eclosão, mas em menores porcentagens. A eclosão só não ocorreu em valores de $\mathrm{pH}$ extremos: $\mathrm{pH} 3$ e 12 .

$\mathrm{Na}$ tabela II são apresentados os resultados de eclosão dos ovos expostos a diferentes concentrações de íns na água. Observa-se que não existem diferenças 
significativas $\left(\Delta_{\text {Tukey }}=1,286 ; \mathrm{p}<0,05\right)$ entre os tratamentos em que se utilizaram água mole, dura, muito dura e moderadamente dura. Entretanto, os resultados dos tratamentos em que se utilizaram água muito mole e mole não fazem parte do mesmo agrupamento de médias, apesar de representarem o ambiente onde o rotífero foi coletado.

Tabela II. Taxa de eclosão (média e erro padrão) dos ovos dormentes de $B$. calyciflorus submetidos a diferentes concentrações de ions na água. As letras $a, b, c$, agrupam as médias significativamente iguais. As médias sem diferença significativa possuem letras iguais.

\begin{tabular}{lcccc}
\hline \multicolumn{1}{c}{ Tipo de água } & $\begin{array}{c}\text { Eclosăo total } \\
(\%)\end{array}$ & Erro padrăo & Variaçăo de $\mathrm{pH}^{*}$ & $\begin{array}{c}\text { Variaçăo da condutividade } \\
\left(\mu \mathrm{S} . \mathrm{cm}^{-1}\right)\end{array}$ \\
\hline Deionizada & $67,5 \mathrm{c}$ & 4,91 & $7,21-8,59$ & $8-108$ \\
Muito mole & $72,5 \mathrm{bc}$ & 3,69 & $6,66-8,28$ & $38-179$ \\
Mole & $87,0 \mathrm{a}$ & 2,19 & $6,72-7,97$ & $137-274$ \\
Moderadamente dura & $83,0 \mathrm{ab}$ & 2,42 & $6,82-8,07$ & $235-449$ \\
Dura & $84,5 \mathrm{ab}$ & 1,70 & $7,40-8,37$ & $400-795$ \\
Muito dura & $84,5 \mathrm{ab}$ & 2,76 & $7,86-8,63$ & $692-1223$ \\
\hline
\end{tabular}

(*) As variações de $\mathrm{pH}$ e condutividade apresentadas representam o menor e o maior valor registrado dentre todos os recipientes de um mesmo tratamento, durante os cinco dias de experimentação.

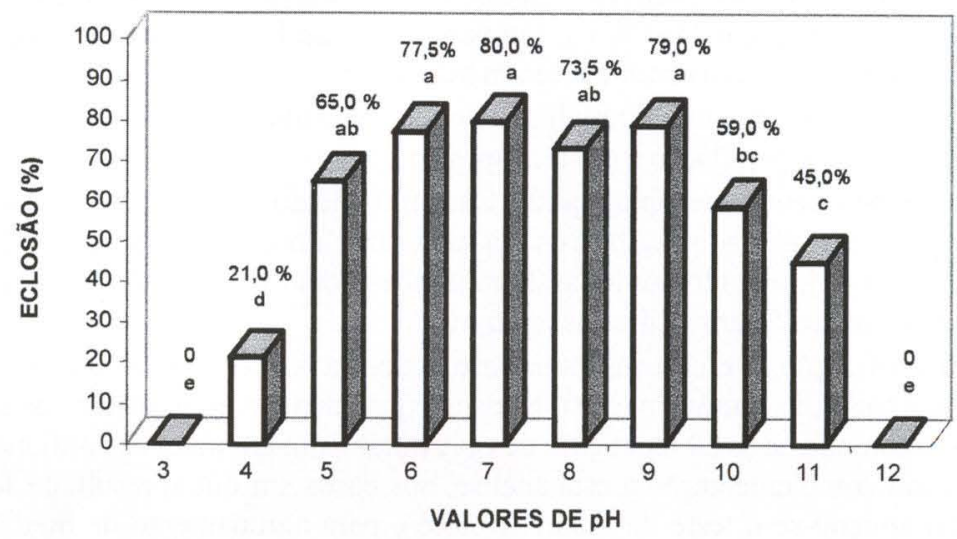

Fig. 1. Taxa de eclosão dos ovos dormentes de $B$. calyciflorus em função de diferentes valores de $\mathrm{pH}$. As letras $\mathrm{a}, \mathrm{b}, \mathrm{c}, \mathrm{d}, \mathrm{e}$, agrupam as médias significativamente iguais. As médias sem diferença significativa possuem letras iguais.

Para os ovos submetidos a diferentes temperaturas observa-se (Tab. III) que não foram detectadas diferenças significativas $(\Delta$ Tukey $=0,974 ; p<0,05)$ entre os resultados obtidos às temperaturas de 25 e $30^{\circ} \mathrm{C}$ (eclosão de 88 e $83 \%$, respectivamente). Fora desta faixa a porcentagem de eclosão decresceu.

Em relação ao fotoperíodo e à intensidade luminosa, os resultados indicam que os ovos dormentes da população estudada eclodem em grandes variações destes parâmetros. Para o fotoperíodo, o resultado do teste de Tukey agrupa os tratamentos em que se utilizaram $8,12,16$ e 24 horas de exposição a luz, e para a intensidade luminosa foram reunidos aqueles em que se empregaram 850, 1350 e 2000 lux (Tab. IV). 
Tabela III. Taxa de eclosão (média e erro padrão) de ovos dormentes de Brachionus calyciflorus submetidos a diferentes temperaturas. As letras $a, b, c$, agrupam as médias significativamente iguais. As médias sem diferença significativa possuem letras iguais.

\begin{tabular}{ccccccc}
\hline \multirow{2}{*}{$\begin{array}{c}\text { Temperatura } \\
\left({ }^{\circ} \mathrm{C}\right)\end{array}$} & \multicolumn{5}{c}{ Eclosăo $(\%)$} & \multirow{2}{*}{ Erro padrão } \\
\cline { 2 - 6 } & Segundo dia & Terceiro dia & Quarto dia & Quinto dia & Total \\
\hline 10 & 0,0 & 0,0 & 0,0 & 0,0 & $0,0 \mathrm{~d}$ & 0,00 \\
15 & 2,5 & 16,0 & 22,0 & 13,5 & $54,0 \mathrm{c}$ & 3,28 \\
20 & 42,0 & 23,0 & 2,0 & 0,0 & $67,0 \mathrm{~b}$ & 3,17 \\
25 & 58,5 & 29,0 & 0,5 & 0,0 & $88,0 \mathrm{a}$ & 2,13 \\
30 & 70,5 & 11,5 & 1,0 & 0,0 & $83,0 \mathrm{a}$ & 2,72 \\
35 & 35,0 & 20,5 & 0,5 & 0,0 & $56,0 \mathrm{C}$ & 2,34 \\
40 & 0,0 & 0,0 & 0,0 & 0,0 & $0,0 \mathrm{~d}$ & 0,00 \\
\hline
\end{tabular}

Tabela IV. Taxa de eclosão (média e erro padrão) dos ovos dormentes de $B$. calyciflorus submetidos a diferentes fotoperiodos e intensidades luminosas. As letras a e b agrupam as médias significativamente iguais. As médias sem diferença significativa possuem letras iguais.

\begin{tabular}{|c|c|c|c|c|c|c|c|}
\hline \multirow{2}{*}{$\begin{array}{l}\text { Fotoperiodo } \\
\text { (Horas) }\end{array}$} & \multirow{2}{*}{$\begin{array}{c}\text { Intensidade } \\
\text { luminosa }(\text { Lux })^{\star *}\end{array}$} & \multicolumn{5}{|c|}{ Eclosão (\%) } & \multirow[t]{2}{*}{ Erro padrão } \\
\hline & & Segundo dia & Terceiro dia & Quarto dia & Quinto dia & Total & \\
\hline 0 & 0 & 42,0 & 8,5 & 1,0 & 0,1 & $52,5 \mathrm{~b}$ & 3,76 \\
\hline 8 & 650 & 33,5 & 22,0 & 3,0 & 0,1 & $59,5 a b$ & 3,36 \\
\hline 12 & 650 & 38,0 & 13,0 & 3,5 & 0,0 & $54,5 \mathrm{ab}$ & 2,76 \\
\hline 16 & 650 & 29,5 & 15,0 & 1,0 & 2,0 & $54,0 a b$ & 3,03 \\
\hline 24 & 650 & 46,0 & 14,5 & 6,5 & 0,0 & $67,0 \mathrm{a}$ & 3,71 \\
\hline 0 & 0 & 19,0 & 43,5 & 1,0 & 0,0 & $64,0 \mathrm{~b}$ & 4,13 \\
\hline 12 & 650 & 46,5 & 18,0 & 4,5 & 0,0 & $69,0 \mathrm{~b}$ & 3,69 \\
\hline 12 & 850 & 61,5 & 21,5 & 4,0 & 0,0 & 87,5 a & 2,28 \\
\hline 12 & 1350 & 78,0 & 8,5 & 0,5 & 0,0 & $87,0 \mathrm{a}$ & 2,19 \\
\hline 12 & 2000 & 62,5 & 26,0 & 0,0 & 0,0 & $88,5 a$ & 1,96 \\
\hline
\end{tabular}

$\left({ }^{\star}\right)$ Fotoperiodo: $\Delta$ Tukey $=1,320 ; p<0,05 ;\left({ }^{\star \star}\right)$ Intensidade Luminosa: $\Delta$ Tukey $=1,177 ; p<0,05$.

Para os experimentos relativos a temperatura, fotoperíodo e intensidade luminosa, os valores de $\mathrm{pH}$ estiveram ao redor da neutralidade (6 a 8) e os de condutividade apresentaram variação semelhante àquela verificada no tratamento em que se utilizou água muito mole do experimento sobre concentração de sais(38 a $\left.179 \mu \mathrm{S} . \mathrm{cm}^{-1}\right)$.

\section{DISCUSSÃO}

Com o procedimento utilizado na alimentação de $B$. calyciflorus procurou-se eliminar a probabilidade de a dieta estar influenciando a viabilidade dos ovos dormentes, pois os organismos, estando suficientemente bem nutridos, devem gerar ovos energeticamente ricos (GILBERT 1980; HaGiwara \& HinO 1990).

A produção de ovos dormentes pelas populações cultivadas foi considerada espontânea e ocorreu sincronicamente durante curtos períodos. Foram testados diferentes fatores de indução da produção destes ovos, mas os resultados positivos foram eventuais, não sendo possivel indicar um parâmetro que, efetivamente, induzisse sua obtenção. Estes testes incluíram o aumento da temperatura da água, em um período de 24 horas, de 20 à $30 \pm 3^{\circ} \mathrm{C}$; a manutenção das populações no escuro e em fotoperíodo de 24 horas e a utilização de intensidades luminosas de 500 e 2000 
lux. Outras tentativas de indução à produção de ovos dormentes foram realizadas com diferentes manejos alimentares relativos a qualidade (oferecimento de cultura unicelular ou uma mistura de espécies de algas) e a quantidade de alimento (grande disponibilidade e interrupção da alimentação). Na prática, as populações foram mantidas em aquários, até o momento em que entravam em intensa e sincronizada produção de ovos. O período entre uma produção de ovos e outra nunca foi o mesmo ou seja, não houve coincidência nos estágios parentais. Futuros trabalhos devem ser desenvolvidos na tentativa de indicar os fatores que induzam a formação de ovos dormentes de rotíferos de regiões tropicais. Estas tentativas são de vital importância, pois o não conhecimento desses fatores constituiu uma das maiores dificuldades para a realização deste trabalho.

\section{Influência do $\mathrm{pH}$}

Durante as 24 horas decorridas entre as trocas de água, verificou-se que em alguns tratamentos ocorreram variações de até duas unidades de $\mathrm{pH}$. Em virtude desta variação empregou-se o termo "exposição dos ovos", e não "manutenção dos ovos" a diferentes valores de $\mathrm{pH}$. A "manutenção" dos ovos em um determinado valor de $\mathrm{pH}$ exigiria a utilização de tampões apropriados, que introduziriam no meio outras variáveis, como teor de sais. Assim, a substituição diária da água, por outra com o pH ajustado, sujeitava os ovos a brusca variação deste parâmetro, principalmente em valores extremos de $\mathrm{pH}$.

Não foram encontradas, na bibliografia consultada, informações sobre a influência do $\mathrm{pH}$ na eclosão de ovos dormentes de rotíferos.

A taxa de eclosão dos ovos dormentes desta espécie, em valores de $\mathrm{pH}$ extremos, demonstra que eles teriam condições de, quando presentes no sedimento dos viveiros de criação de peixes, resistir aos processos de calagem. Estes procedimentos são comumente utilizados em aqüicultura, e causam grandes variações de $\mathrm{pH}$ da água. Além disso estes ovos também seriam capazes de resistir às acentuadas alterações de $\mathrm{pH}$ e condutividade da água observadas diariamente, principalmente quando a água dos viveiros está eutrofizada, permanecer viáveis e produzir alguns jovens que recolonizariam o ambiente.

Como não foram observadas diferenças significativas de taxa de eclosão, entre os valores de $\mathrm{pH}$ de 5 a 9 , é provável que a variação verificada nos valores de condutividade entre estes tratamentos $\left(86 \mathrm{a} 830 \mu \mathrm{S} . \mathrm{cm}^{-1}\right)$, não tenha influenciado o resultado obtido.

\section{Influência da concentração de íons}

Durante as tentativas de cultivo de $B$. calyciflorus foi empregado meio de cultura artificial, proposto por GILBERT (1970). No presente trabalho, este procedimento não apresentou resultados satisfatórios, provavelmente por ter sido, originalmente, desenvolvido para populações de regiões temperadas do hemisfério norte onde, em geral, as águas possuem maiores concentrações de íons. Este fato, demonstrando a necessidade de se desenvolver um meio de cultura para populações de regiões tropicais, em que as águas são menos "duras", motivou a realização deste experimento. 
Apesar de não existirem diferenças significativas entre os resultados dos tratamentos em que se utilizaram água mole, moderadamente dura, dura e muito dura, observou-se maior atividade dos organismos que eclodiram nos tratamentos com água muito mole e mole. Nos demais tratamentos, o comportamento do rotífero não foi "normal". No tratamento com água deionizada, por exemplo, o rotífero ficava praticamente parado, isto é, não apresentava os movimentos normais de natação observados nos tratamentos com água muito mole e mole. Já, nos tratamentos com água dura e muito dura, após o nascimento apresentavam comportamento aparentemente normal mas, quando deixados no recipiente de eclosão, morriam após algumas horas.

Como foi constatado no experimento anterior, em que os ovos de B. calyciflorus eclodiram satisfatoriamente na faixa de $\mathrm{pH}$ de 5 a 9 , as diferenças entre os valores de $\mathrm{pH}$, observadas neste experimento, provavelmente não influíram nos resultados obtidos. Quanto à variação da condutividade, os resultados demonstram que os ovos dormentes deste rotífero, apesar de necessitarem de quantidades mínimas, eclodem em diferentes concentrações dos íons utilizados.

Os valores de $\mathrm{pH}$ e as concentrações de sais, que proporcionaram os melhores resultados de eclosão de ovos nos respectivos experimentos anteriores, indicam que, quando o embrião entrou em contato com o meio externo, seu sistema osmorregulador teve a capacidade de executar várias compensações, que permitiram o término do desenvolvimento embrionário e o nascimento do jovem rotífero.

\section{Influência da temperatura}

Na literatura encontram-se referências a diferentes valores de temperatura, que proporcionam bons resultados de eclosão de ovos dormentes de rotíferos do gênero Brachionus Pallas, 1776 e que, normalmente, são semelhantes aos do ambiente em que vive a população. POURRIOT et al. (1981) observaram eclosão de ovos de B. rubens Ehrenberg, 1838 nas temperaturas de 15 e $19^{\circ} \mathrm{C}$, com taxas de eclosão de 70 e $90 \%$, respectivamente. Para B. calyciflorus, $85 \%$ dos ovos do clone Cr79 eclodiram na temperatura de $5^{\circ} \mathrm{C}$ e $100 \%$, na de $14^{\circ} \mathrm{C}$, enquanto para os do clone SR80, nos mesmos valores de temperatura, as taxas foram 8,7 e $51,6 \%$, respectivamente (POURRIOT et al. 1982). Quanto aos ovos do rotífero marinho $B$. plicatilis Müller, 1786 MINKOFF et al. (1983) observaram que a eclosão ocorre em uma faixa de temperatura de $10^{\circ} \mathrm{C}\left(68 \%\right.$ de eclosão) a $30^{\circ} \mathrm{C}(6 \%$ de eclosão) e que os ovos submetidos a certas temperaturas eclodem após dois dias de incubação, como também foi observado neste trabalho. Da mesma forma, para a população de B. calyciflorus estudada, os melhores resultados de eclosão de ovos foram registrados em valores de temperatura que comumente ocorrem no ambiente natural da espécie $\left(88 \%\right.$ de eclosão a $25^{\circ} \mathrm{C}$ e $83 \%$ a $\left.30^{\circ} \mathrm{C}\right)$. Verificou-se eclosão destes ovos às temperaturas de 35 e $15^{\circ} \mathrm{C}$ ( 56 e $54 \%$, respectivamente). Em valores mais extremos (temperaturas de 10 e $40^{\circ} \mathrm{C}$ ) não se observou eclosão.

\section{Influência do fotoperíodo e da intensidade luminosa}

Relativamente ao fotoperíodo, POURRIOT et al. (1981) e BLANCHOT \& POURRIOT (1982b) estudaram a influência deste parâmetro (fotoperíodos de $0,8 \mathrm{e}$ 16 horas) sobre os ovos de B. rubens e B. plicatilis, respectivamente. Estes autores 
observaram que, quando os ovos são expostos a fotoperíodo (8 ou 16 horas) e temperatura adequada, pode ocorrer taxa de eclosão entre 70 e $100 \%$ mas, na ausência de luz, não ocorre eclosão de ovos de nenhuma das espécies. De maneira semelhante, POURRIOT et al. (1982) utilizaram ovos de dois clones de B. calyciflorus cultivados no escuro ou com fotoperíodo de oito horas. Os autores observaram para o clone $\mathrm{Cr} 79$ que, quando produzidos por populações cultivadas no escuro, $100 \%$ dos ovos eclodem na presença ou ausência de luz e, para o clone SR80, quando cultivado com fotoperíodo de 8 horas, $84,6 \%$ dos ovos eclodem na presença de luz, e $57,1 \%$, no escuro. Neste trabalho, em que B. calyciflorus foi cultivado com fotoperíodo de 14 horas, obtiveram-se taxas de eclosão de $59,5 \%$ para os ovos submetidos a fotoperíodo de oito horas e $52,5 \%$ para os ovos mantidos no escuro. Isto demonstra que a necessidade de luz para eclosão de ovos de repouso também é característica intrínseca das populações estudadas, pois o fotoperíodo adequado promoveu melhores resultados de eclosão, não sendo, entretanto, fundamental.

Não foram encontrados na literatura pertinente trabalhos relacionando eclosão de ovos dormentes de Brachionus a diferentes intensidades luminosas. Resultados interessantes, no entanto, foram apresentados por BLANCHOT \& POURRIOT (1982a), estudando eclosão dos ovos de B. rubens em função de diferentes comprimentos de onda do espectro visível (branco/62\% de eclosão; azul/ $62 \%$; verde/ $12 \%$; amarelo/20\% e vermelho/2\%), concluindo que a fotossensibilidade máxima dos embriões desta espécie está situada entre comprimentos de onda de 400 a $480 \mathrm{~nm}$. Estes autores estudaram a influência da qualidade da luz e não da intensidade. Entretanto, os resultados obtidos neste trabalho, para os ovos de B. calyciflorus expostos a diferentes intensidades da luz branca, demonstram que este parâmetro também é importante para ativação de ovos de rotíferos.

Observa-se que o fotoperíodo e a intensidade luminosa, quando utilizados de maneira adequada, promovem melhores resultados de eclosão total e em um período de tempo menor. Assim, verifica-se um número maior de rotíferos eclodindo no segundo dia de manutenção dos ovos, à medida que o fotoperíodo e a intensidade luminosa aumentam.

Para as condições em que todos os experimentos foram realizados, os melhores resultados de eclosão dos ovos dormentes do rotífero $B$. calyciflorus foram obtidos com valores de $\mathrm{pH}$ da água de 5-9, concentração iônica de sais selecionados de 220 a $880 \mathrm{mg} \cdot \mathrm{l}^{-1}$, temperatura de $25-30^{\circ} \mathrm{C}$, fotoperíodo igual ou superior a oito horas por dia e intensidade luminosa de 850 a 2000 lux.

AGRADECIMENTO. A Coordenadoria de Capacitação e Aperfeiçoamento de Pessoal do Ensino Superior (CAPES), pela concessão de bolsa de pós-graduação, a nível de mestrado.

\section{REFERÊNCIAS BIBLIOGRÁFICAS}

American Public Health Association. 1989. Standard methods for the examination of water and wastewater. New York, $17^{\text {th }}$ ed., VIII $+16 \mathrm{p}$.

BLANCHOT, J. \& R. POURRIOT. 1982a. Effets de l'intensité d'éclairement et de la longueur d'onde sur l'éclosion des oeufs de durée de Brachionus rubens (Rotifère). Note. C.R. Acad. Sc. Paris 295 (3): 123-125. 
1982b. Influence de trois facteurs de l'environnement, lumière, température et salinité, sur l'éclosion des oeufs de durée d'un clone de Brachionus plicatilis (O.F. Müller) Rotifère. Note. C.R. Acad. Sc. Paris 295 (III): 243-246. GILBERT, J.J. 1970. Monoxenic cultivation of the rotifer Brachionus calyciflorus in a defined medium. Oecologia 4: 89-101.

. 1974 Dormancy in rotifers. Trans. Amer. Microsc. Soc. 93: 490-513.

1980. Female polymorphism and sexual reproduction in the rotifer $A s-$ planchna: evolution and their relationship and control by dietary alpha-tocopherol. Amer. Nat. 116: 409-431.

GuILLARD, R.R.L. \& C.J. LoRENZEN. 1972. Yellow-green algae with chlorophyllid-c. Jour. Phycol. 8 (1): 10-14.

Hagiwara, A. 1994. Practical use of rotifer cysts. Isr. Jour. Aquac., Bamidgeh, 46 (1): 13-21.

HagiwarA, A. \& A. HiNO. 1990. Feeding history and hatching of resting eggs in the marine rotifer Brachionus plicatilis. Bull. Jap. Soc. Sci. Fish. 56 (12): 1965-1971.

KING, C.E. 1980. The genetic structure of zooplancton populations, p.315-328. In: W.C. KERFOOT (Ed.). Evolution and ecology of zooplancton communities. Hanover, New England Univ. Press.

MinKofF, G.; E. LUBZENS \& D. KAHAN. 1983. Environmental factors affecting hatching of rotifer (Brachionus plicatilis) resting eggs. Hydrobiol. 104: 61-69.

Portella, M.C; M.A.. Cestarolli; J.R. Verani \& N.E.T. Rojas. 1997. Produção de organismos planctônicos para alimentação inicial de larvas de peixes de água doce. Bol. Inst. Pesca 24: 79-89.

Pourriot, R.; C. Rougier \& D. BENEST. 1981. Rôle de la lumière et de la température dans l'éclosion des oeufs de durée de Brachionus rubens Ehr. (Rotifère). Neth. Jour. Zool. 31 (4): 637-649.

Pourriot, R.; D. BENEST \& C. Rougier. 1982. Processus d'éclosion des oeufs de durée de Brachionus calyciflorus Pallas (Rotifère) comparaison de deux clones. Vie Milieu 32 (2): 83-87.

PourRiot, R. \& T.W. SNELL. 1983. Resting eggs in rotifers. Hydrobiol. 104: 213-224.

SNEDECoR, G.W. \& W.G. Cochran. 1971. Statistical Methods. Iowa, Iowa State University Press Ames, 593p.

Recebido em 16.VII.1998; aceito em 16.XI.1999. 